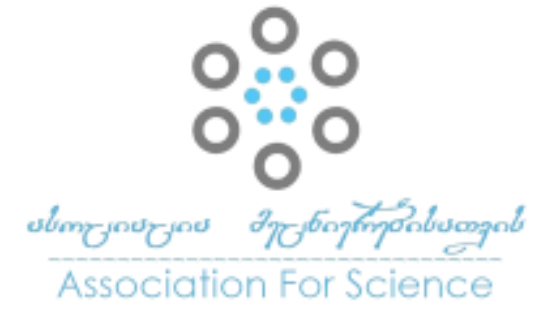

\section{Georgian Scientists}

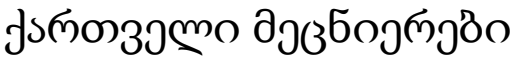

Vol. 4 Issue 1, 2022

https://doi.org/10.52340/gs.2022.04.01.04

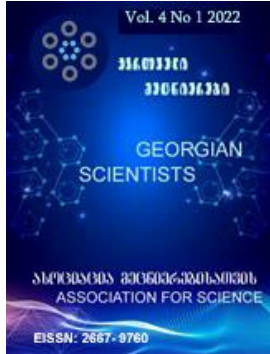

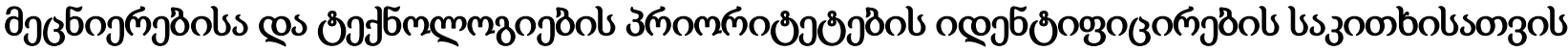

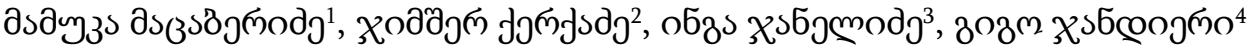

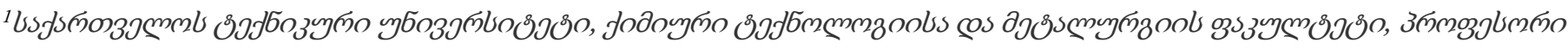

Orcid ID: https://orcid.org/0000-0002-3228-1447

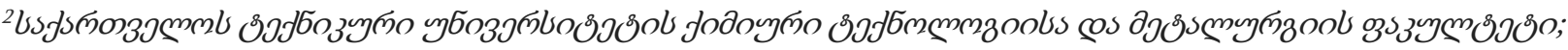

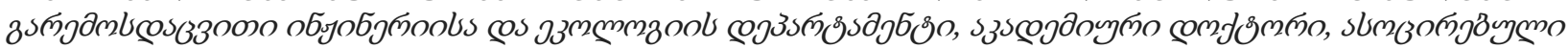

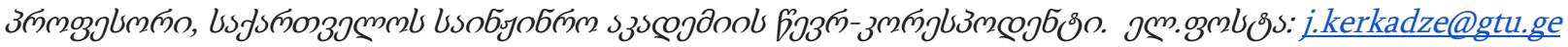

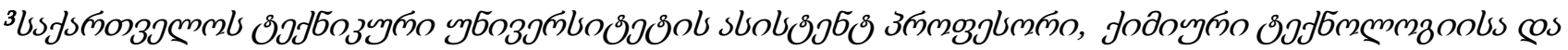

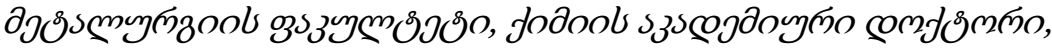

\section{E-mail: i.janelidze@gtu.ge; Orcid ID: https://orcid.org/0000-0002-9961-7638}

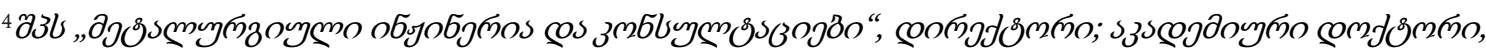
JM-ozmlogs: gigo.jandieri@gmail.com Orcid ID: https://orcid.org/0000-0003-2976-1201

\section{8ับขว}

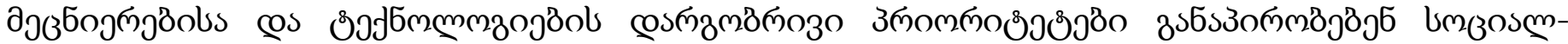

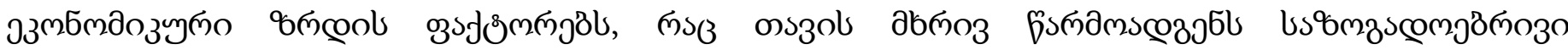

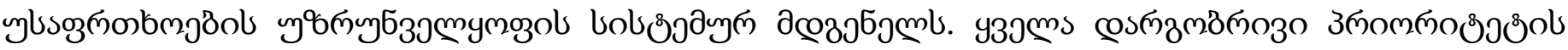

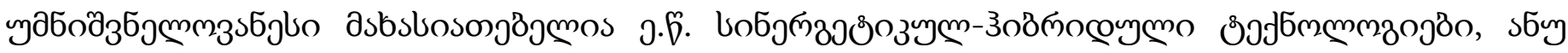

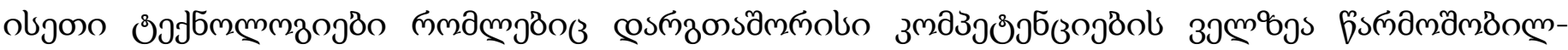

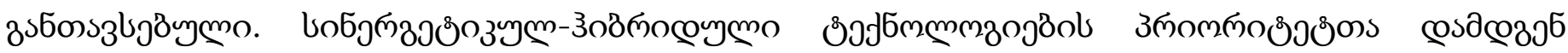

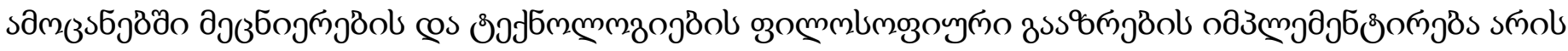

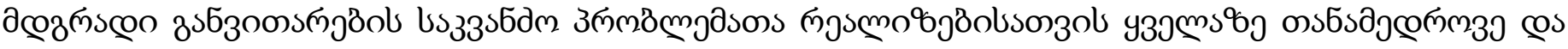

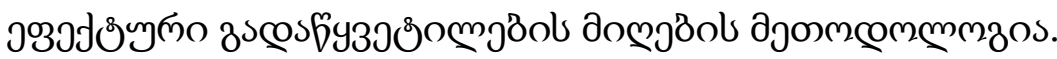

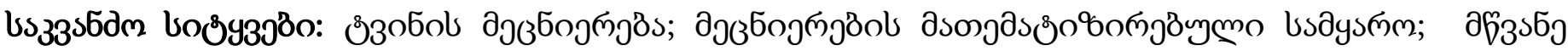

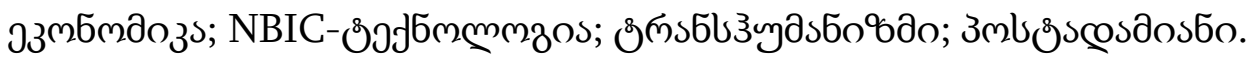




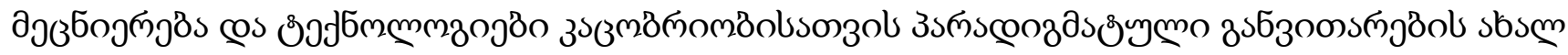

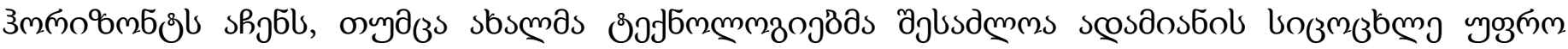

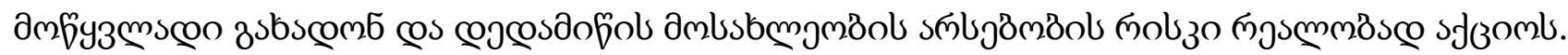

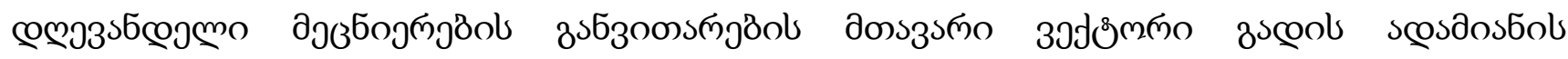

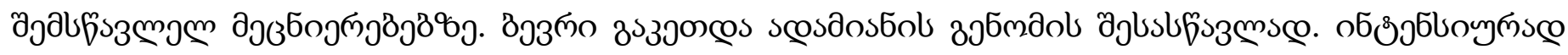

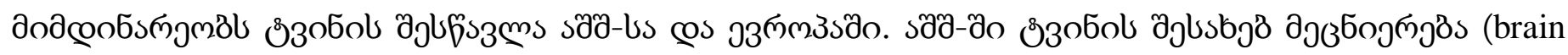

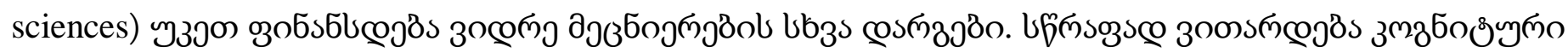

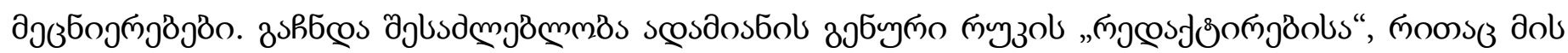

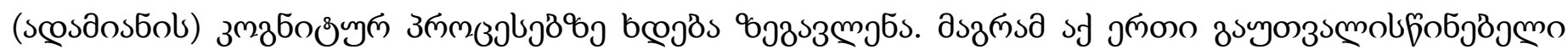

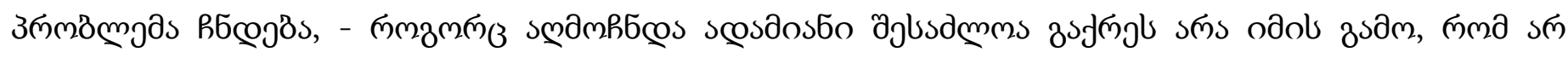

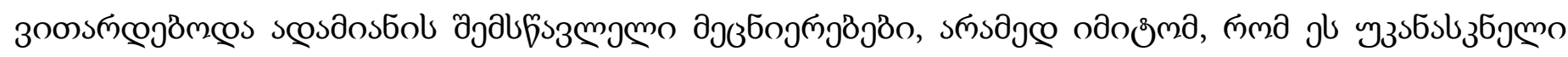

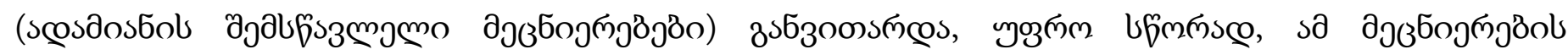

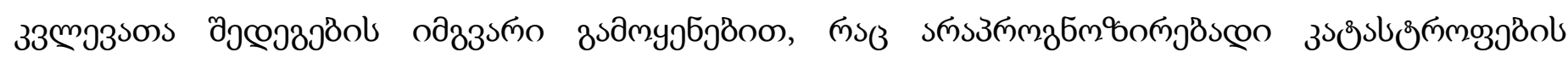

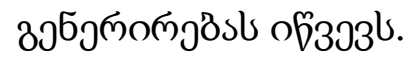

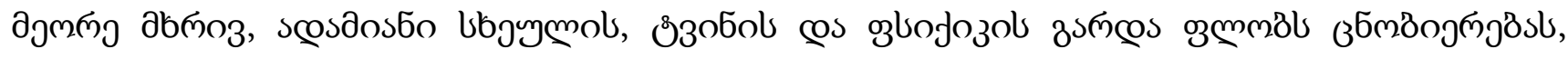

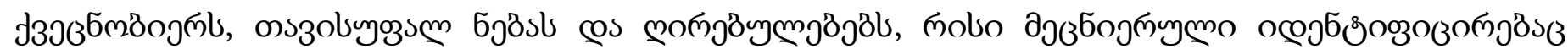

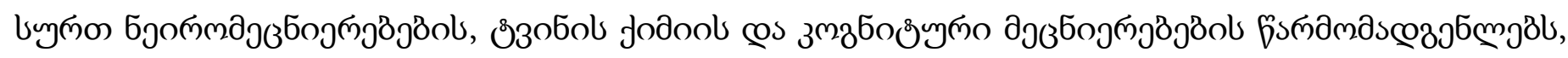

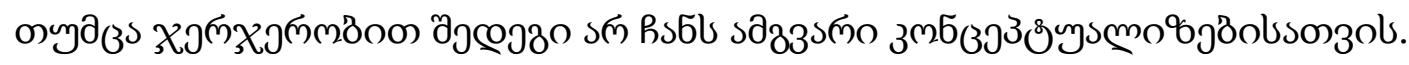

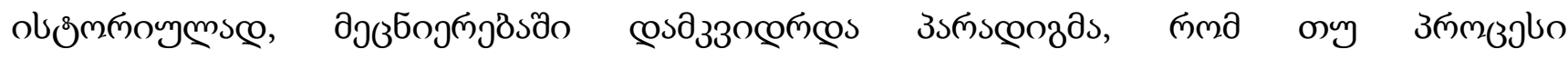

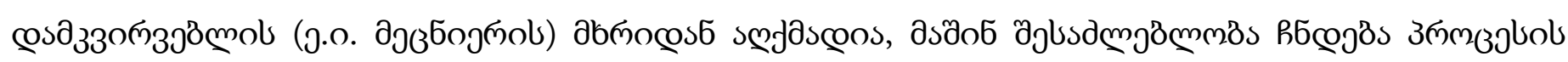

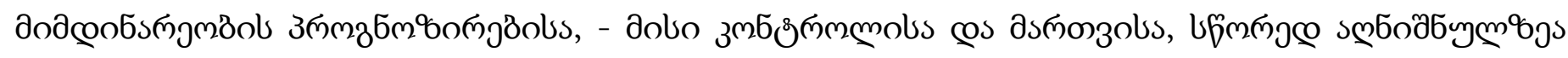

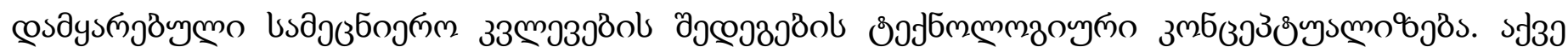

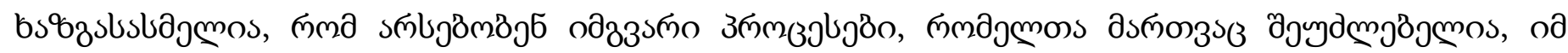

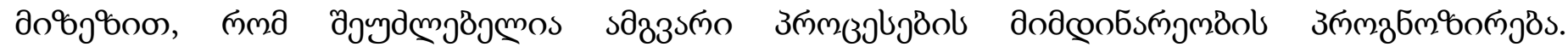

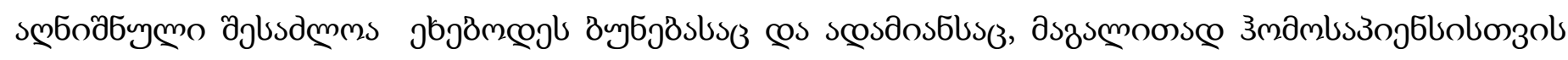

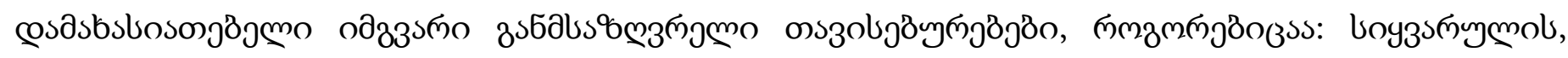

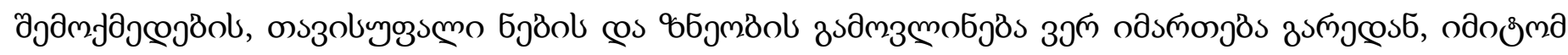

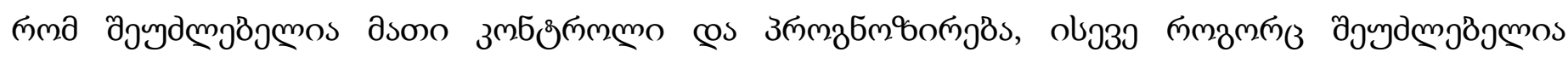




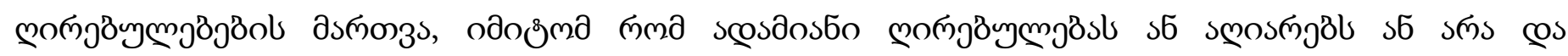

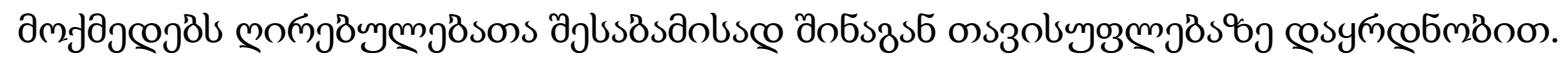

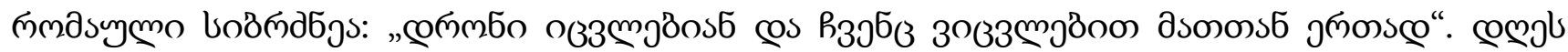

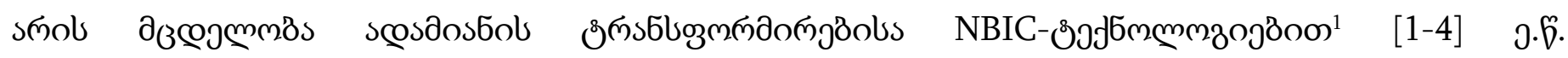

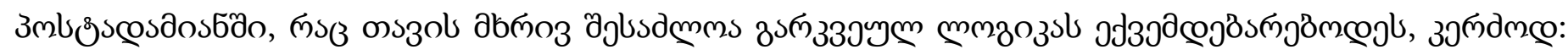

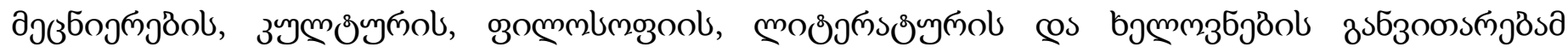

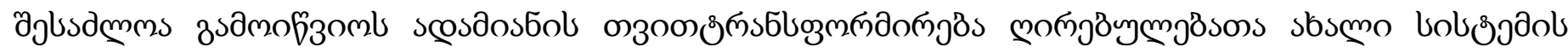

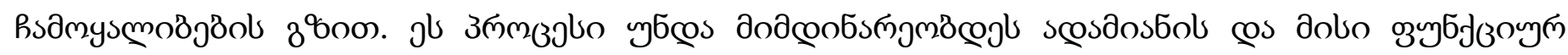

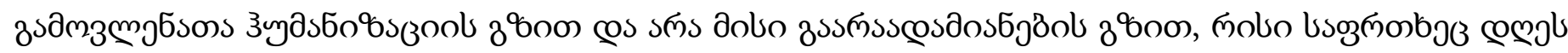

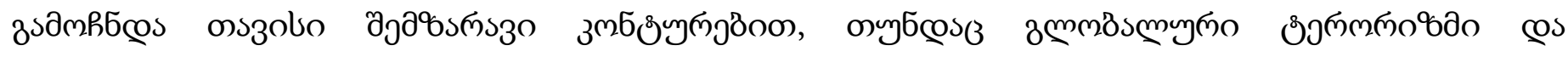

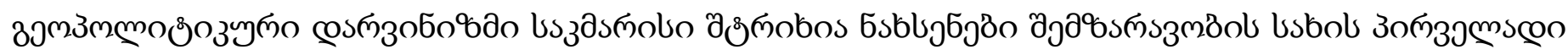
sмfaobsoz̧ol.

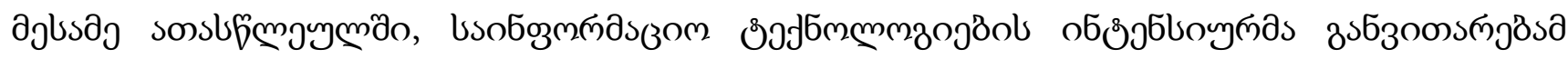

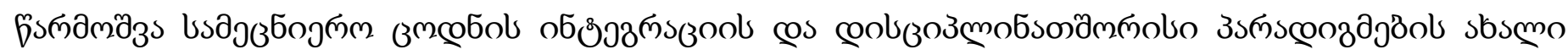

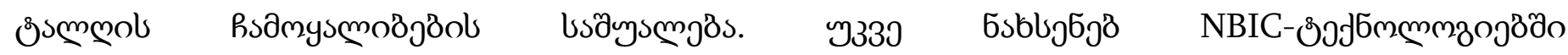

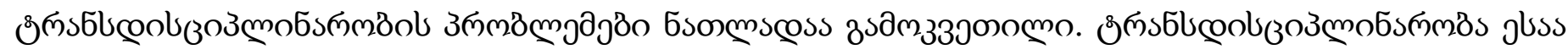

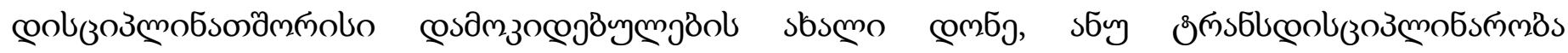

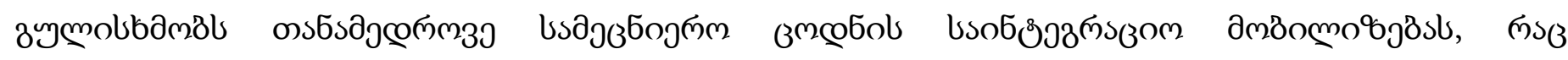

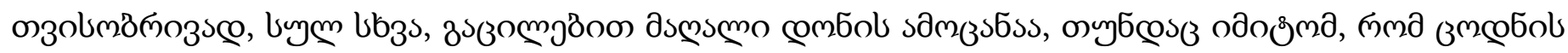

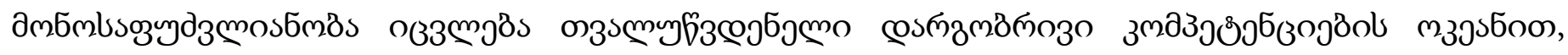

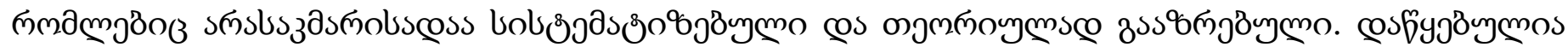

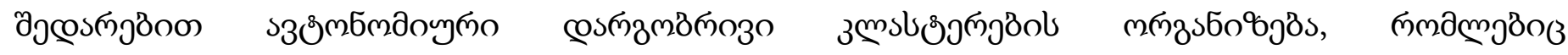

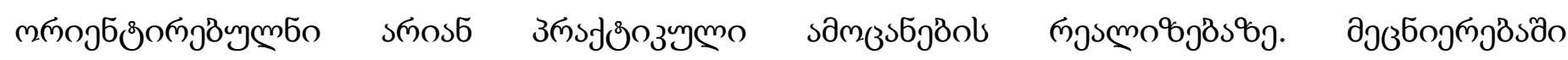

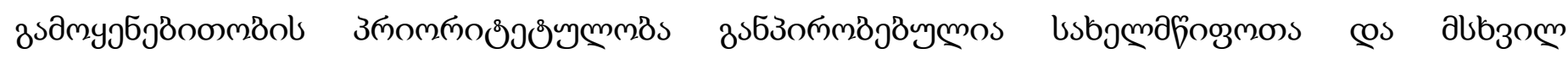

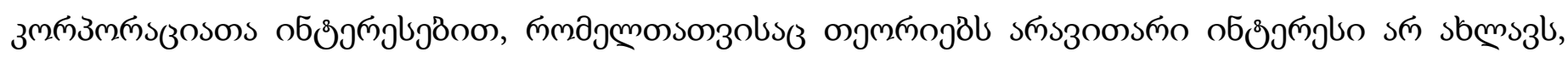

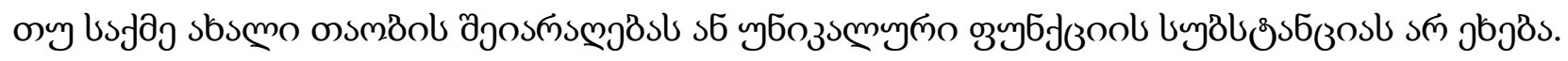

${ }^{1}$ NBIC (Nano-, Bio-, Information technology and Cognitive science) oీ

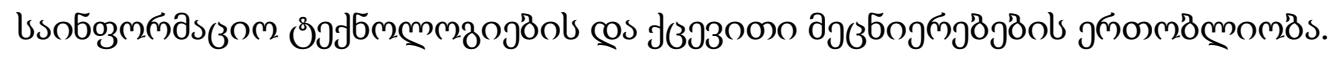




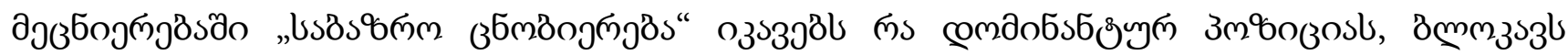

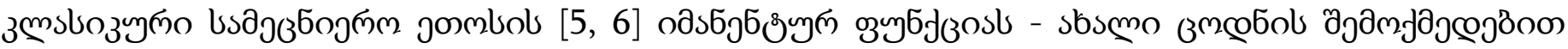

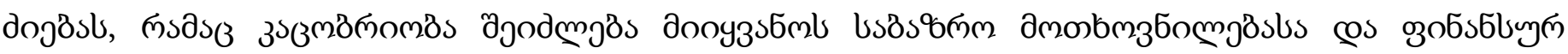

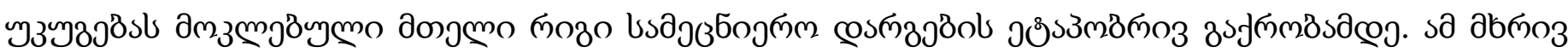

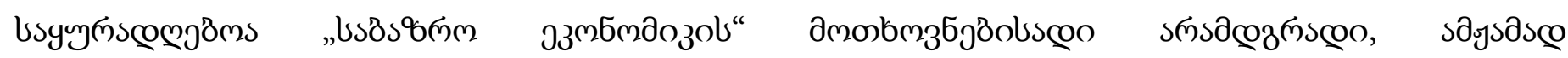

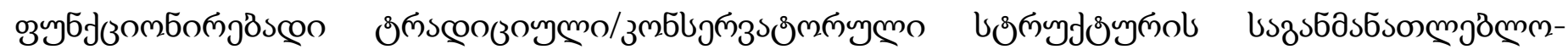

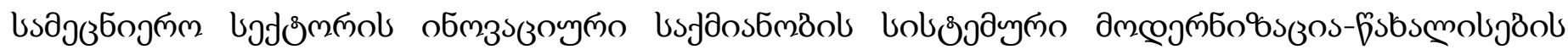

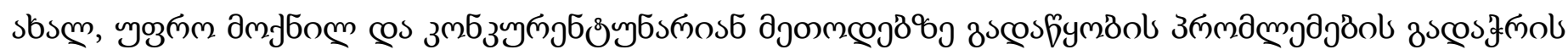

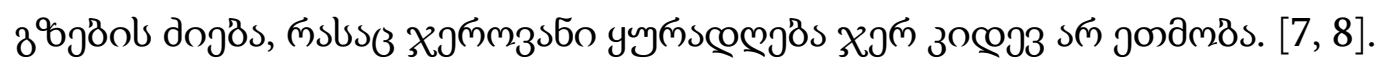

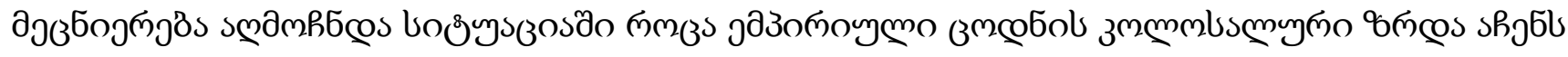

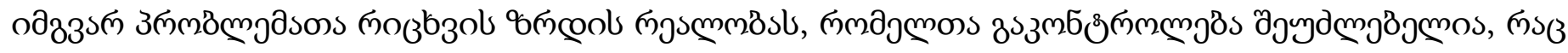

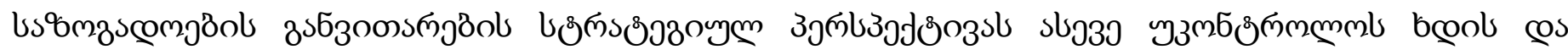

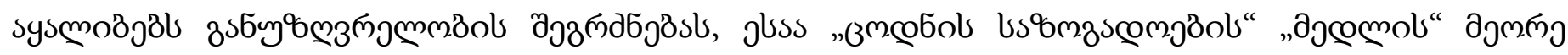

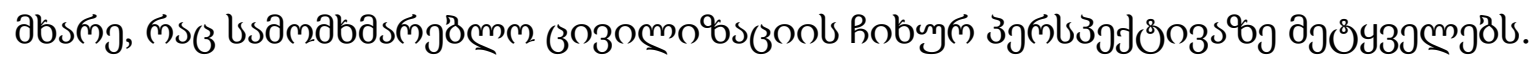

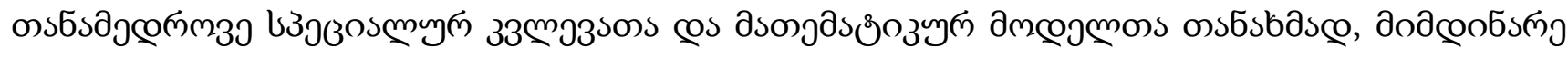

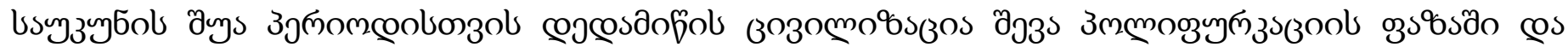

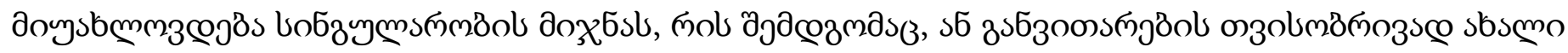

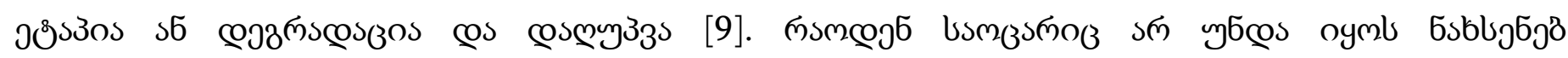

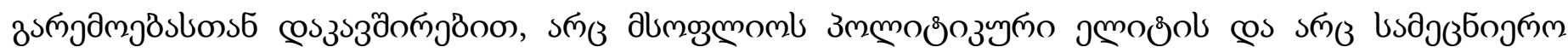

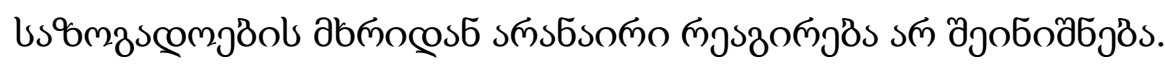

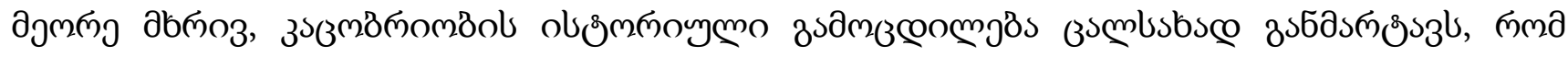

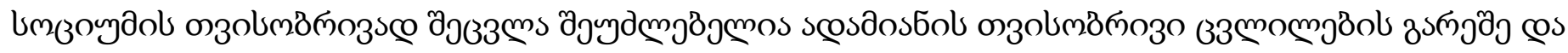

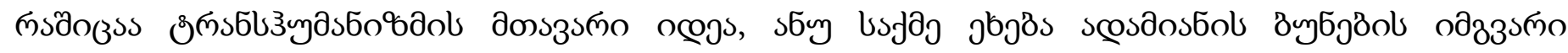

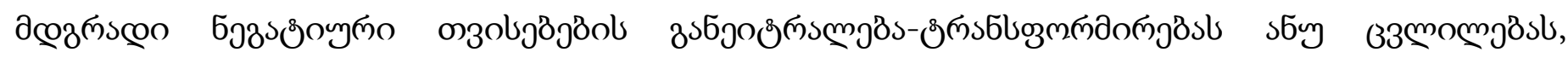

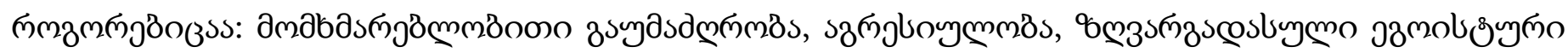

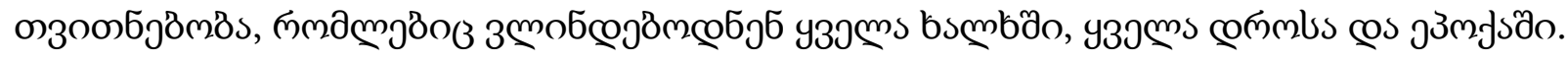

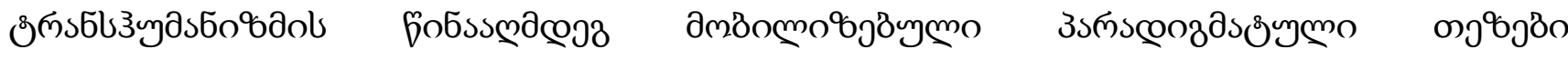

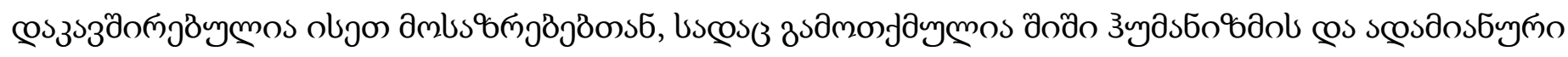

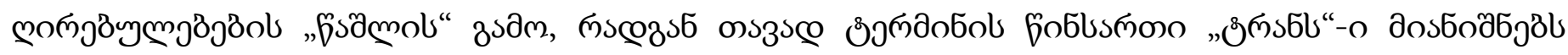

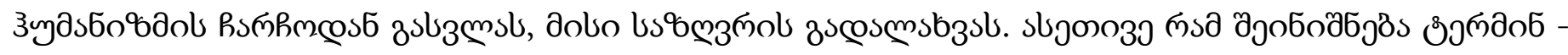




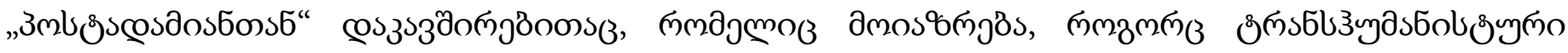

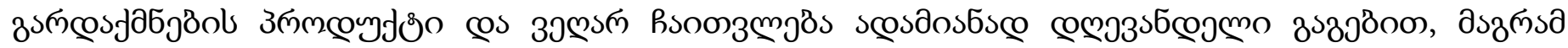

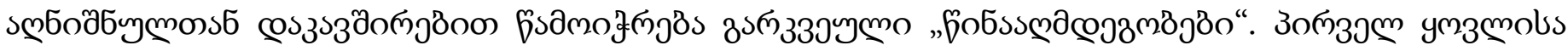
costus\%ylo8

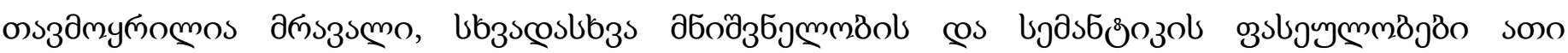

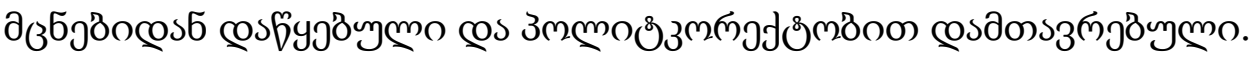

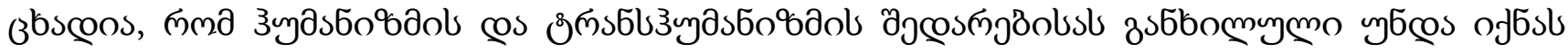

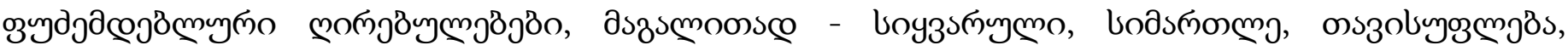

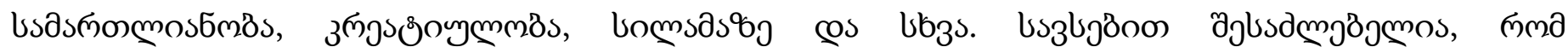

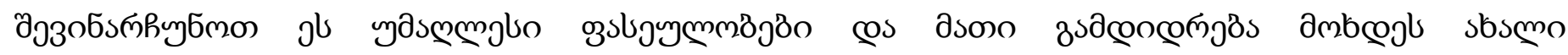

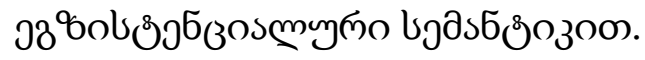

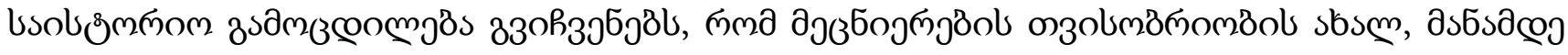

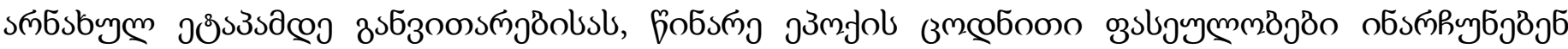

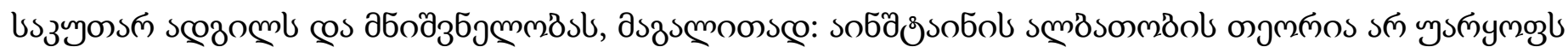

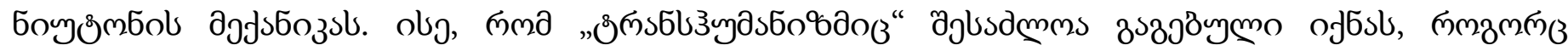

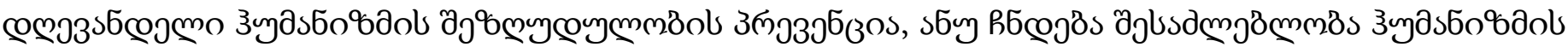

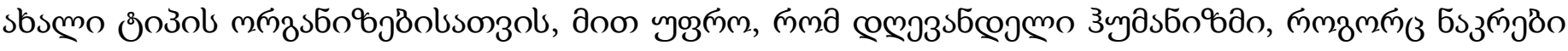

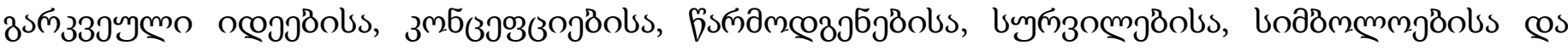

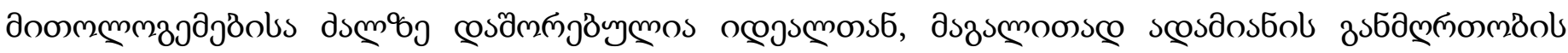
ocgstronst.

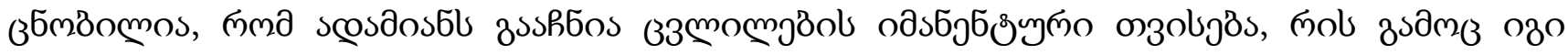

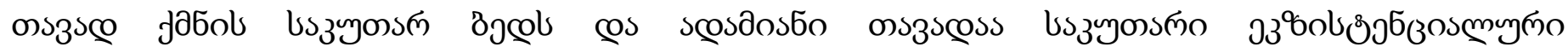

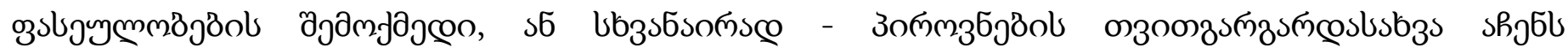

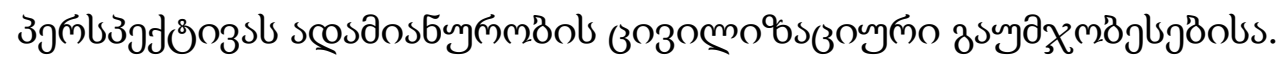

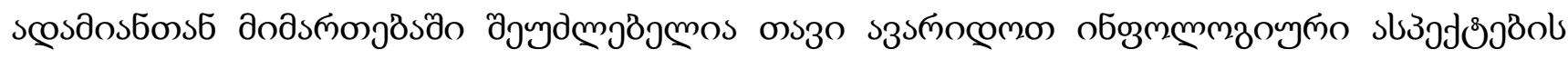

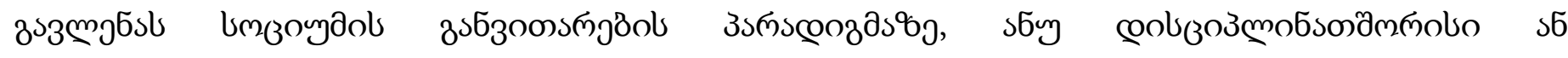

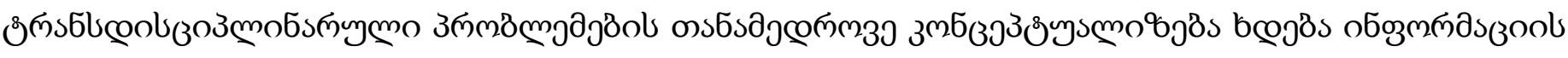

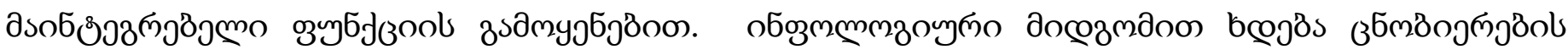

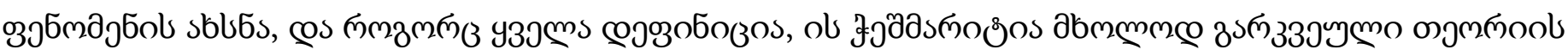

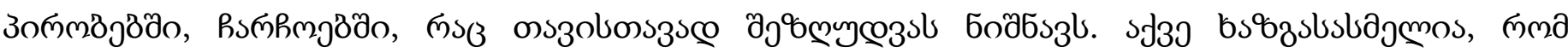




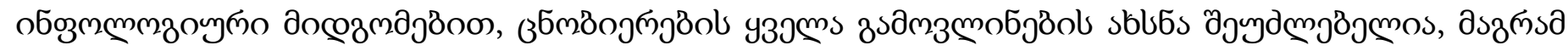

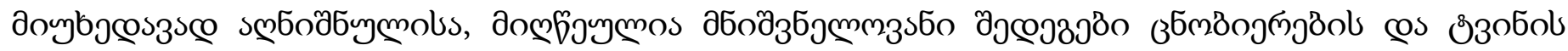

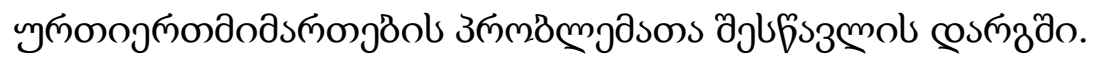

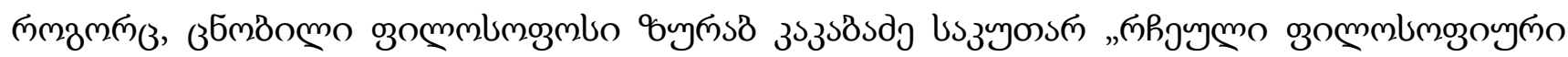

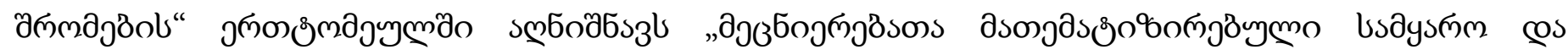

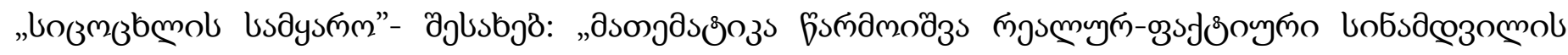

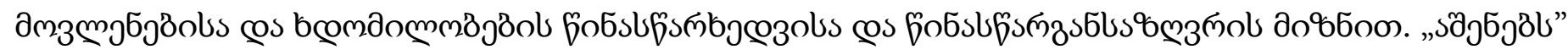

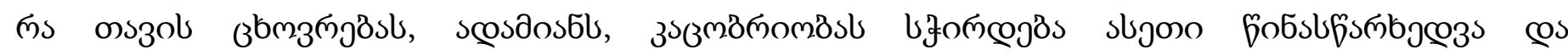

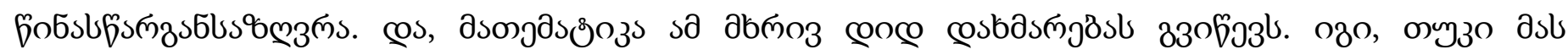

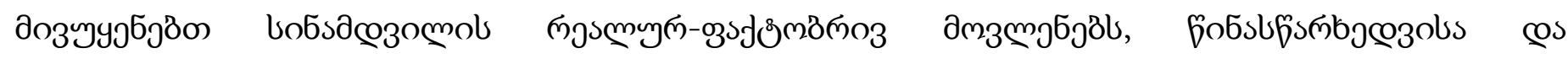

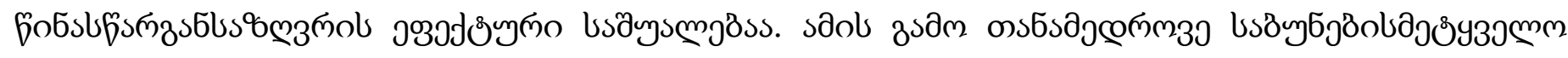

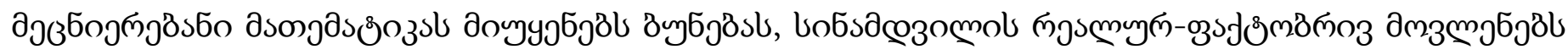

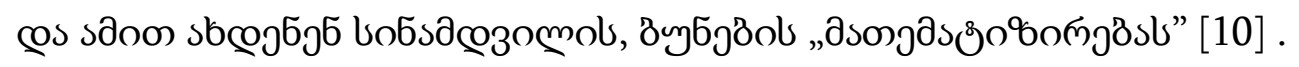

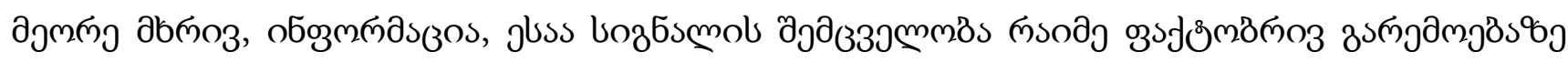

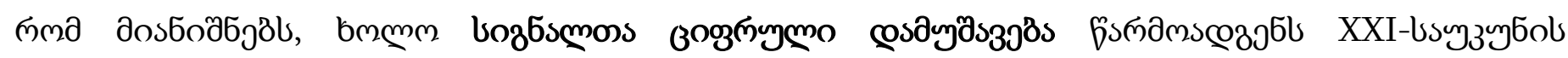

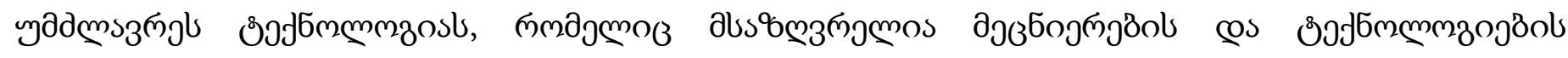

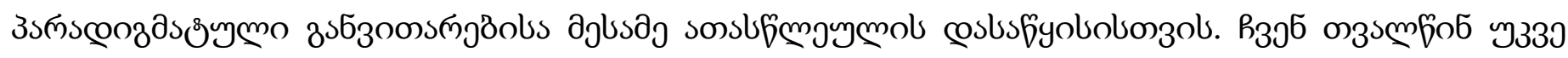

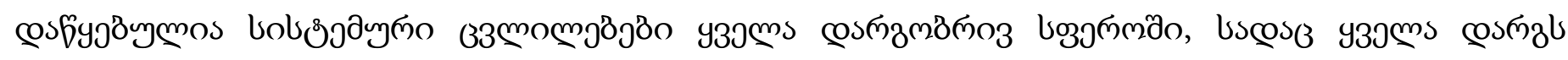

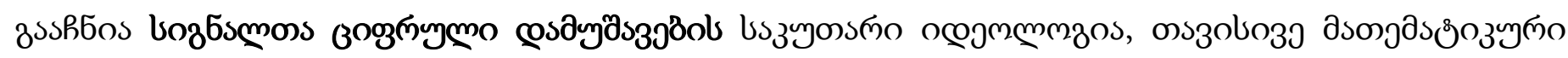

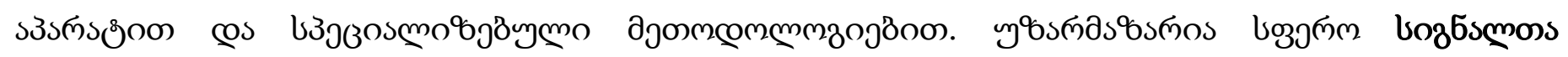

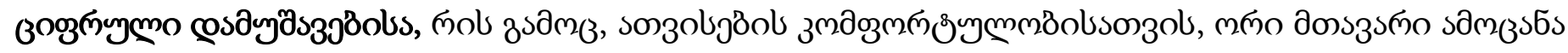

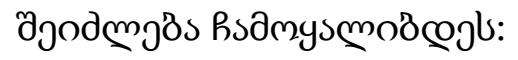

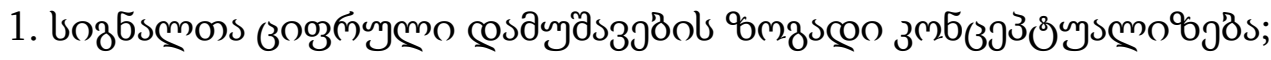

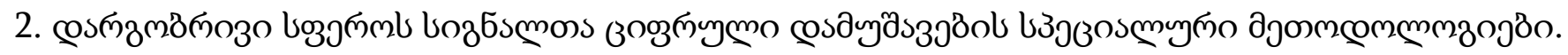

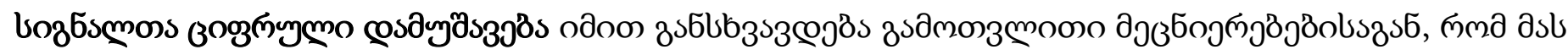

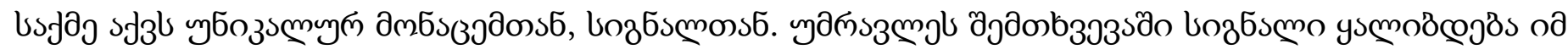

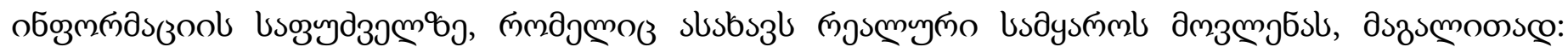

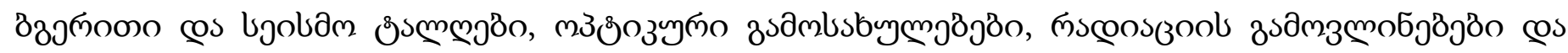




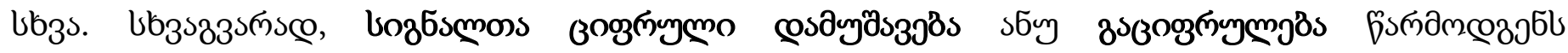

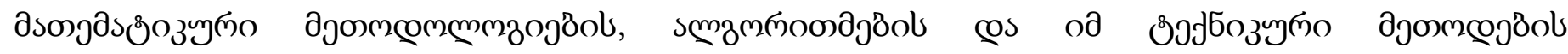

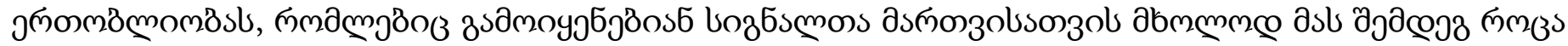

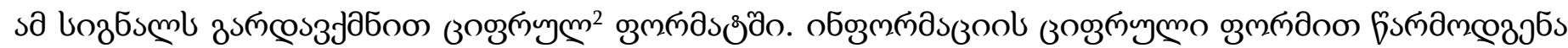

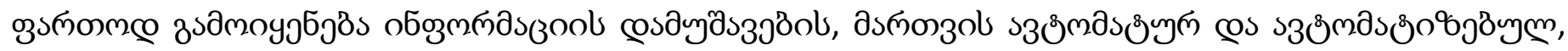

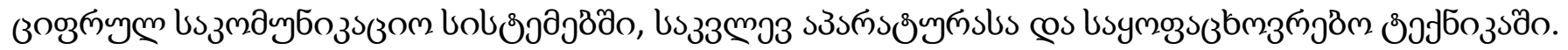

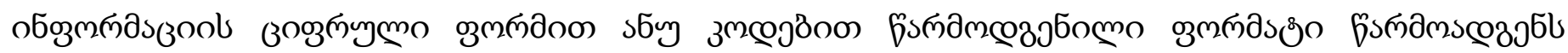

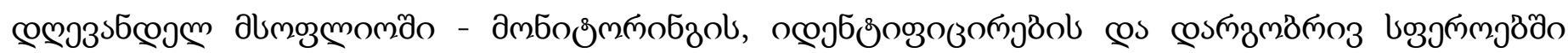

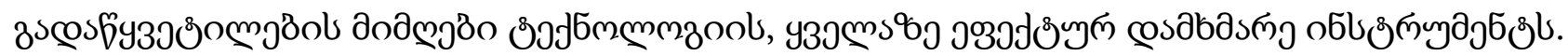

\section{cosb336jöolsonzol}

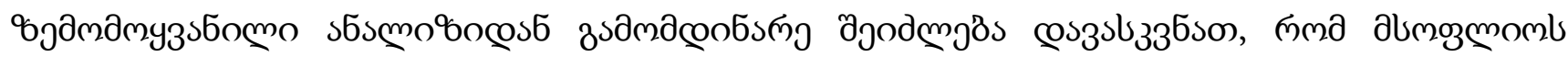

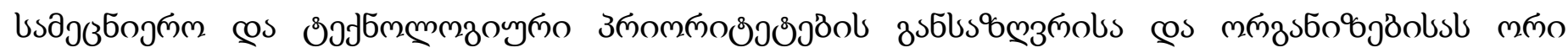

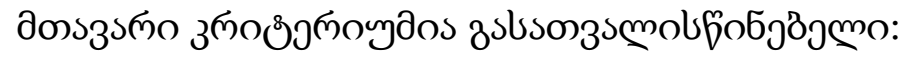

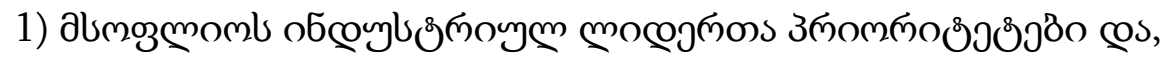

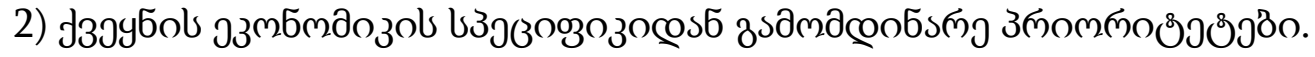

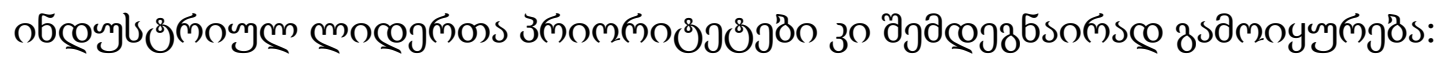

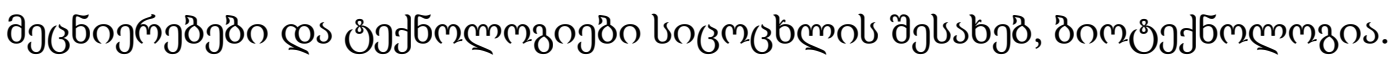

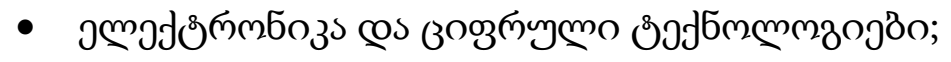

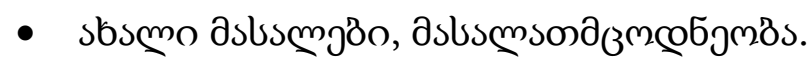

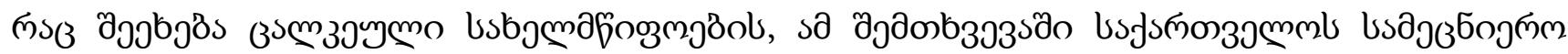

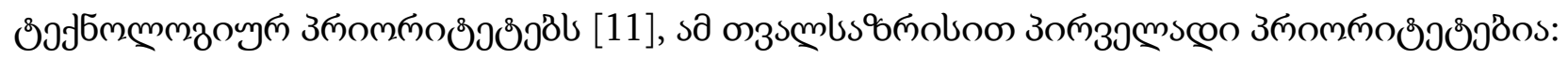

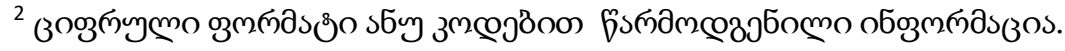




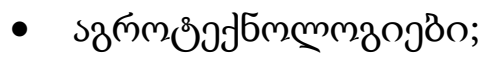

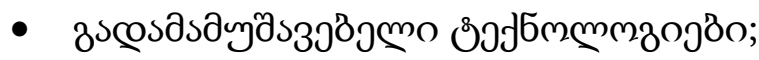

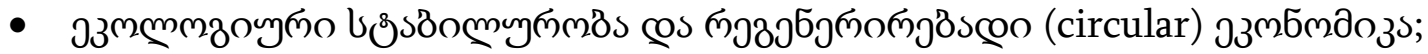

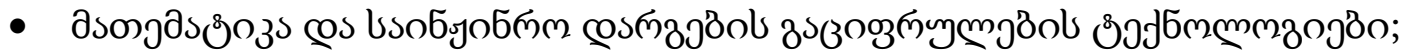

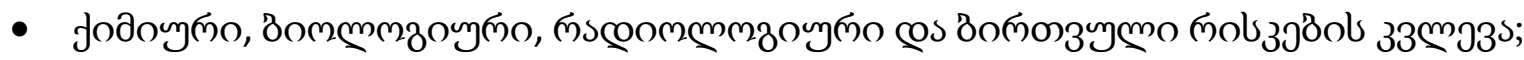

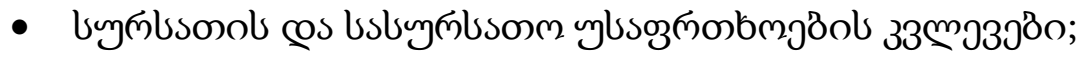

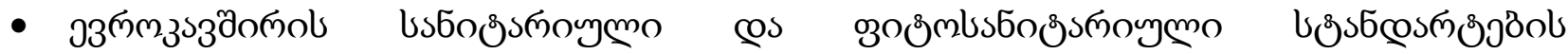

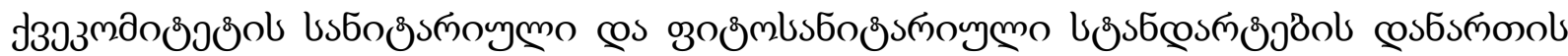

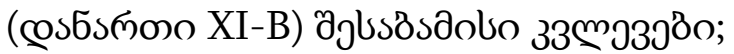

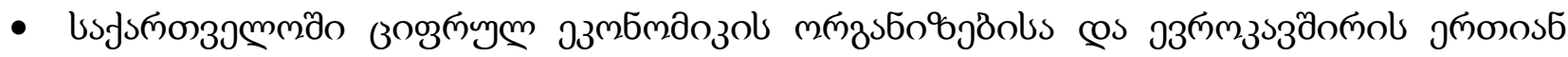

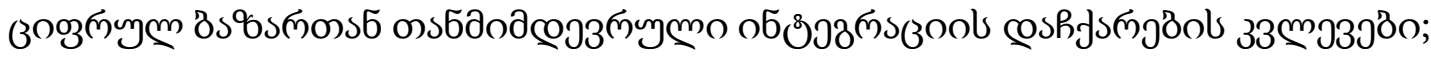

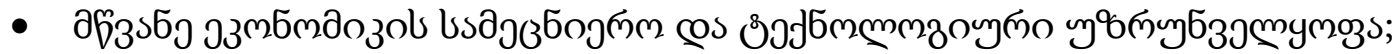

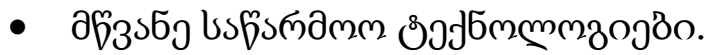

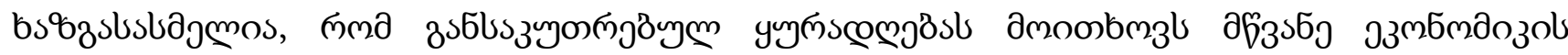

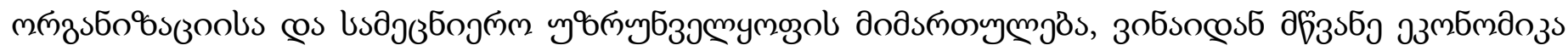

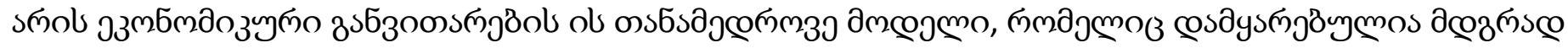

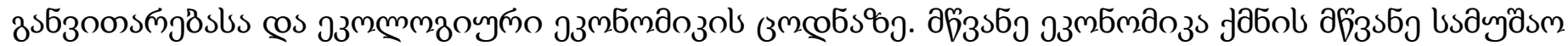

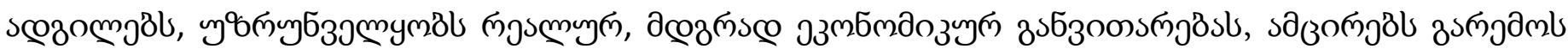

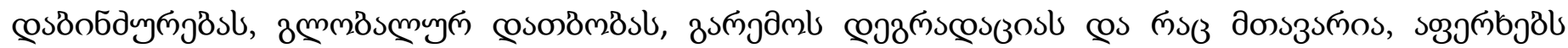

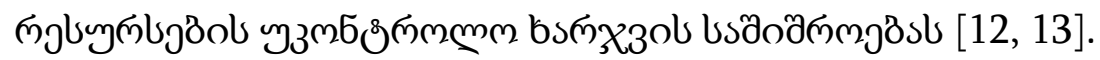

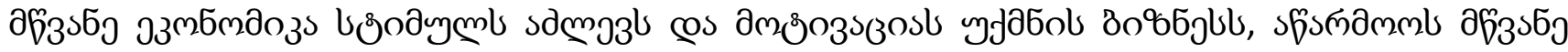

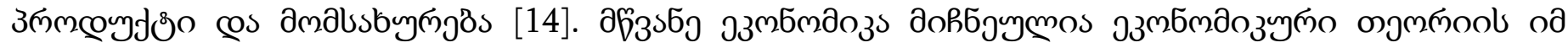
змазмбјббіs

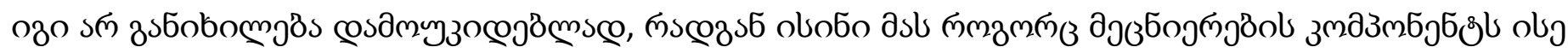

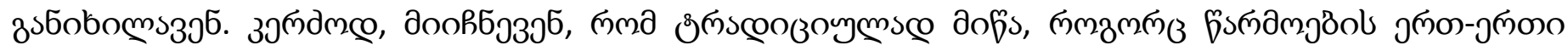

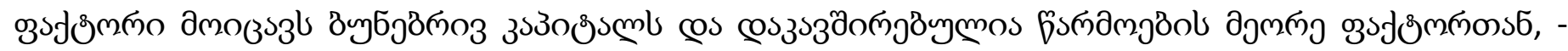

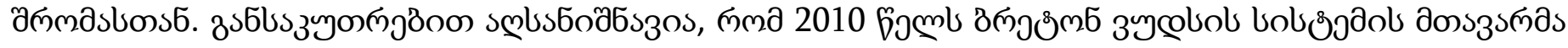

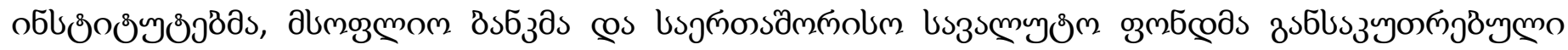

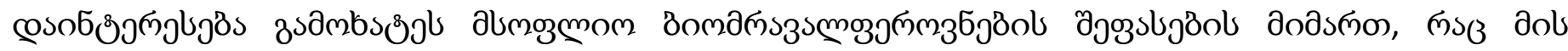

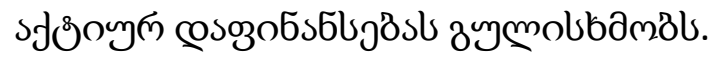




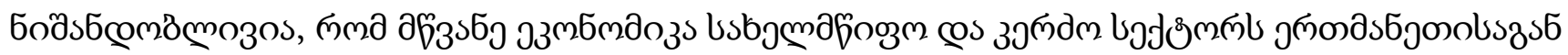

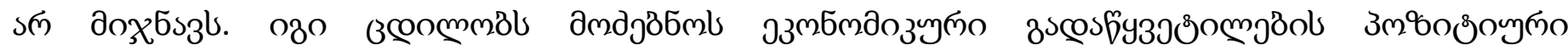

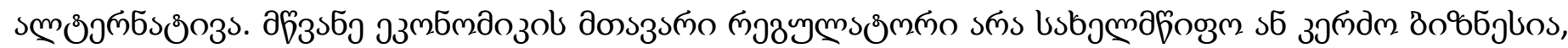

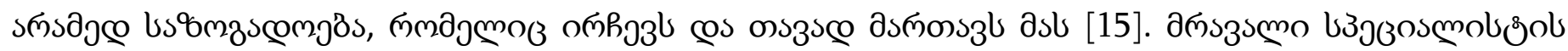

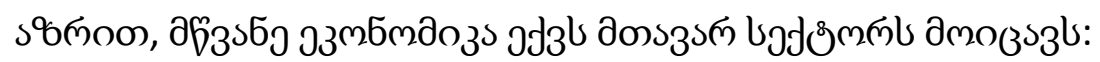

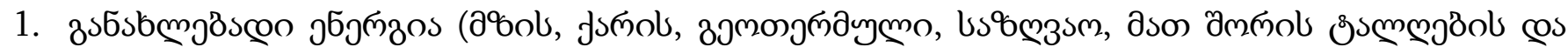

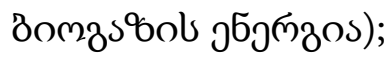

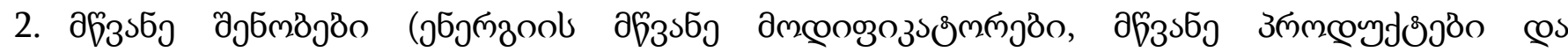

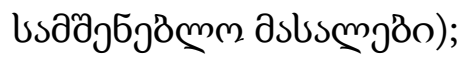

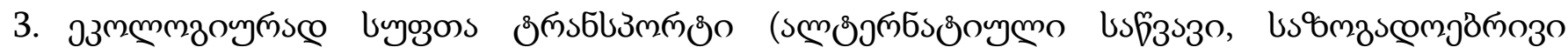

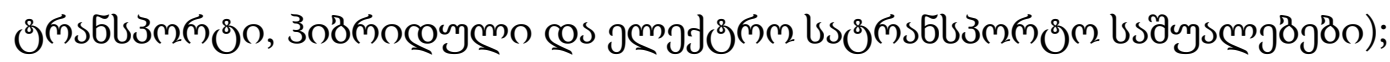

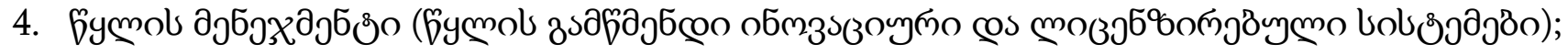

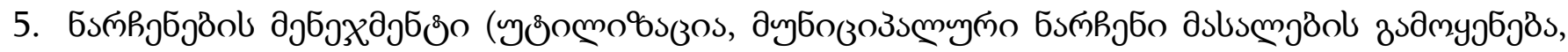

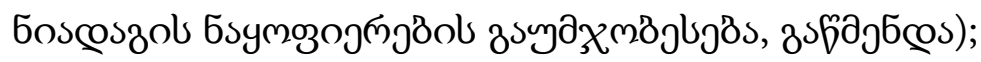

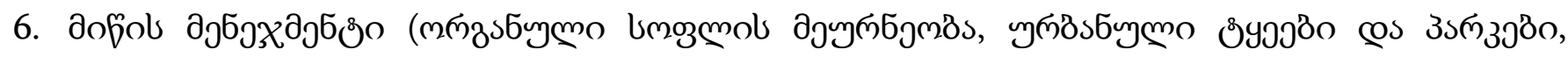

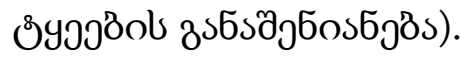

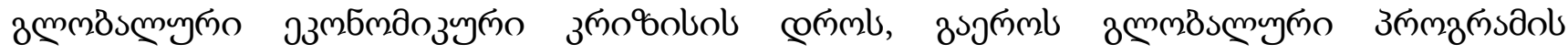

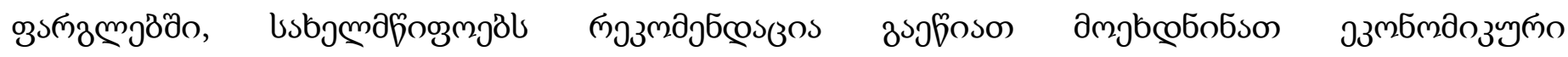

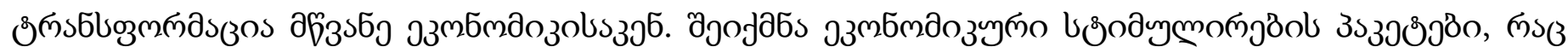

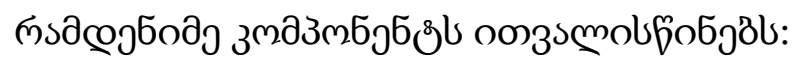

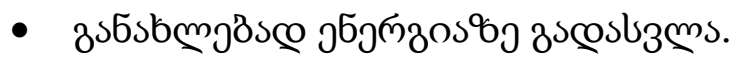

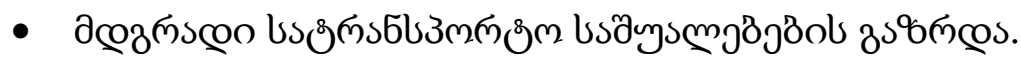

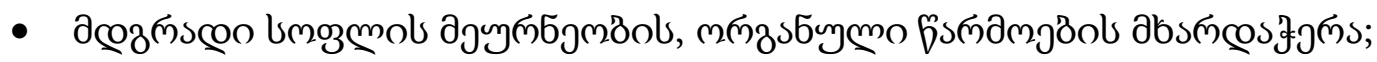

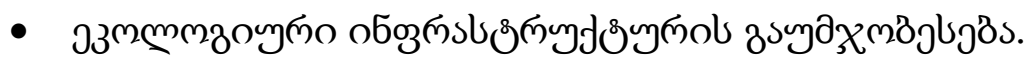

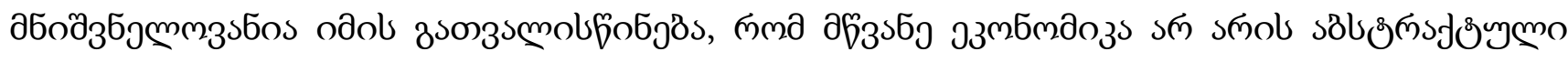

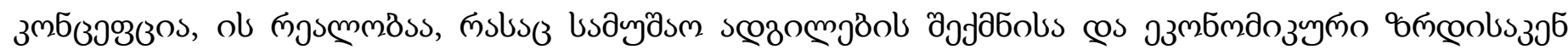

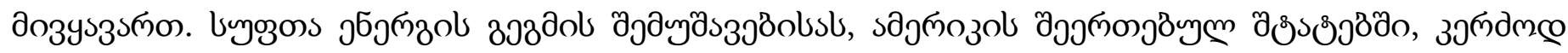




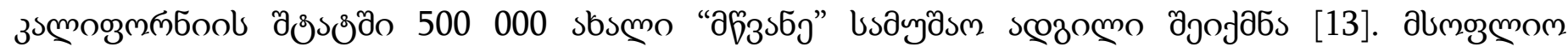

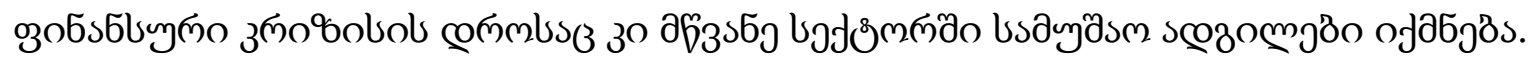

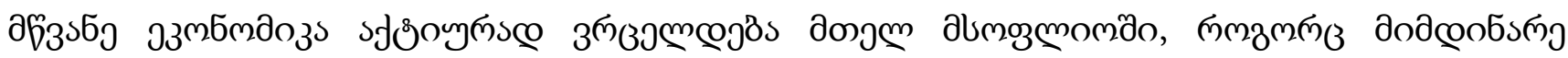

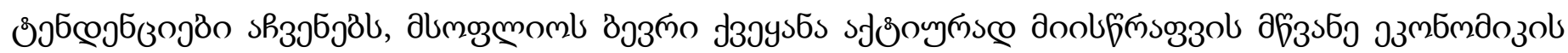

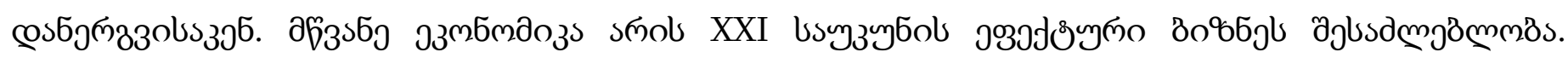

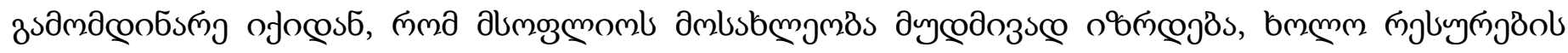

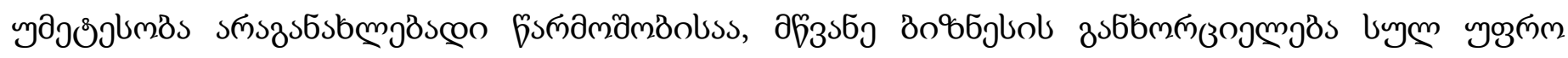

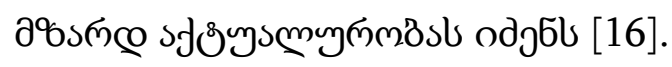

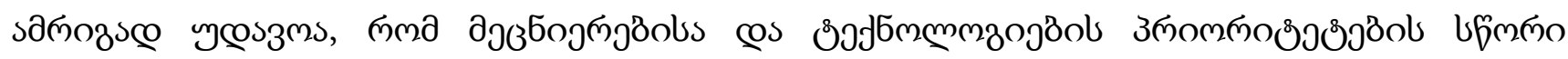

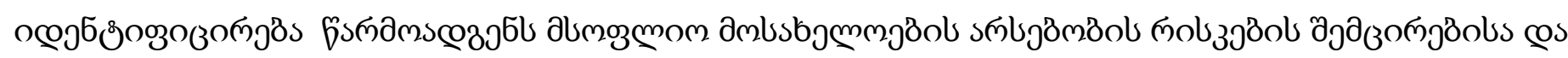

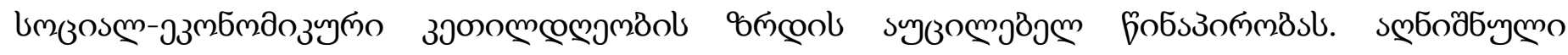

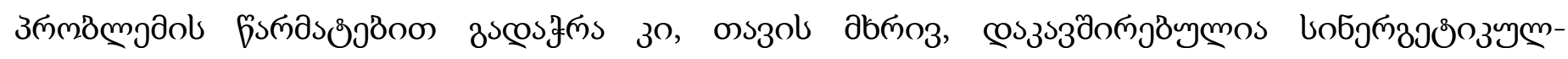

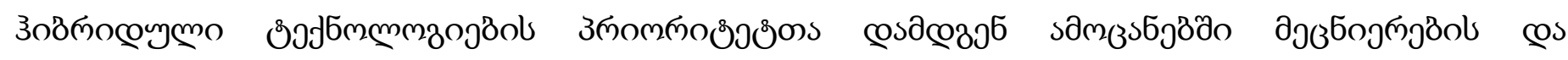

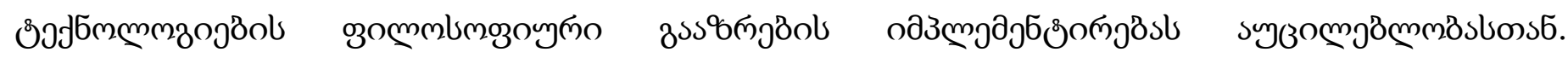

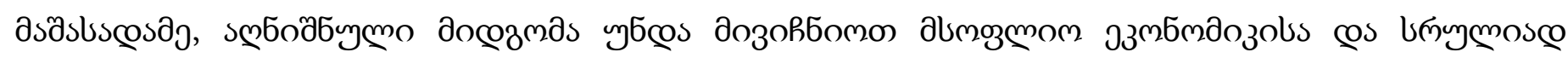

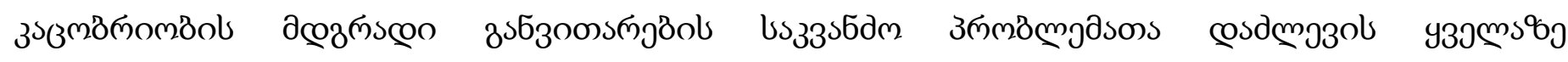

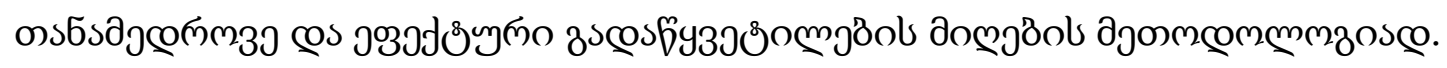




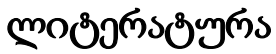

1. Converging Technologies for Improving Human Performance Nanotechnology, Biotechnology, Information Technology and Cognitive Science. Edited by Mihail C. Roco and William Sims Bainbridge National Science Foundation Kluwer Academic Publishers (currently Springer), Dordrecht, The Netherlands, 2003, 482 p.

2. I. Matyushenko, Dr. Sc. of Economics, Professor, O. Azarenkova, PhD. Department of International Economic Relations, School of International Economic Relations and Travel Business, V.N. Karazin Kharkiv National University, Ukraine. Organic Combination of Converging Nbic-Technologies and Smart temp Advanced Technologies of Industry 4.0. International Scientific Journal "Industry 4.0", 2019, Year IV, Issue 1, pp. 11-14

3. M.C. Roco and W.S. Bainbridge. Converging technologies for improving human performance: nanotechnology, biotechnology, information technology and cognitive science. Kluwer, 2002.

4. James Canton Ph.D. Designing the Future. NBIC Technologies and Human Performance Enhancemen. Institute for Global Futures, San Francisco, California 94123, USA. URL: https://pdfs.semanticscholar.org/9b19/ae6ecd6ae66edc85b257d5e902dcd9838217.pdf

5. R.K. Merton. The Institutional Imperatives of Science. Sociology of Science; Ed. B. Barnes. L.: Penguin Books, 1972; P. 65-79; Merton R.K. The Sociology of Science. Chicago: Chicago University Press, 1973, p.p. 267-278

6. R.K. Merton. The Ambivalence of Scientists. Science and Society; Ed. N. Kaplan. Chicago: Rand McNally, 1965.

7. Глобальное будущее 2045. Антропологический кризис. 2013, стр. 353. https://www.hse.ru/data/2015/03/07/1315916450/\%D0\%93\%D0\%BB\%D0\%BE\%D0\%B1\%D0\%B0\%D 0\%BB\%D1\%8C\%D0\%BD\%D0\%BE\%D0\%B5\%20\%D0\%B1\%D1\%83\%D0\%B4\%D1\%83\%D1\%89\%D 0\%B5\%D0\%B5\%202045.\%20\%D0\%90\%D0\%BD\%D1\%82\%D1\%80\%D0\%BE\%D0\%BF\%D0\%BE\%D 0\%BB\%D0\%BE\%D0\%B3\%D0\%B8\%D1\%87\%D0\%B5\%D1\%81\%D0\%BA\%D0\%B8\%D0\%B9\%20\%D 0\%BA\%D1\%80\%D0\%B8\%D0\%B7\%D0\%B8\%D1\%81.pdf

8. I. Janelidze, G. Jandieri, M. Matsaberidze. To a general analysis of the problems of the development of Georgian universities: a brief description and justification of ways to solve them. Conference: International Scientific-Practical Conference - CSASR 2021: "System «Science - Technologies - 
Innovations»: Methodology, Experience, Perspective"At: Minsk, BelarusVolume: https://center.basnet.by/wp-content/uploads/2021/07/conference programme 2021.pdf

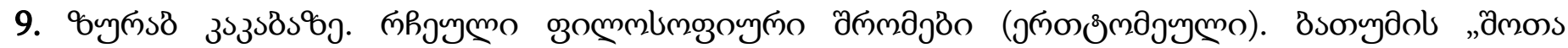

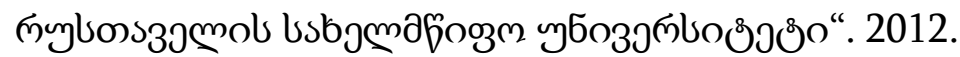

10. Digital Signal Processing. A Practical Guide for Engineers and Scientists by Steven W. Smith. 2011. $720 \mathrm{p}$.

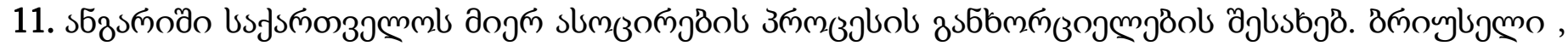
9 бмэдљэณо 2017.

12. G. Jandieri, O. Portyanko. Recycling - a modern solution for improving the operational efficiency of the mining and metallurgical enterprises. International Conference "The Development of Mining and Geology is the Precondition for the Revival of Economy”, Book of Abstracts, 2021, pp. 42-46 URL: http://msgeorgia2012.com/files/SUL\%20BOLO\%20TEZISEBI2021.pdf\#page=42

13. G. Jandieri, I. Janelidze. On Climate Change Mitigation Measures in Ferrous and Non-Ferrous Metallurgy (General Analysis). International Scientific Conference „Natural Disasters in the 21st Century: Monitoring, Prevention, Mitigation“. Proceedings, ISBN 978-9941-491-52-8, Tbilisi, Georgia, December 20-22, 2021. Publish House of Iv. Javakhishvili Tbilisi State University, Tbilisi, 2021, pp. 42 - 45. URL: http://openlibrary.ge/bitstream/123456789/9574/1/12 Conf ND 2021.pdf

14. G. Jandieri. A generalized model for assessing and intensifying the recycling of metal-bearing industrial waste: A new approach to the resource policy of manganese industry in Georgia, Resources Policy, Volume 75, 2022, https://doi.org/10.1016/j.resourpol.2021.102462

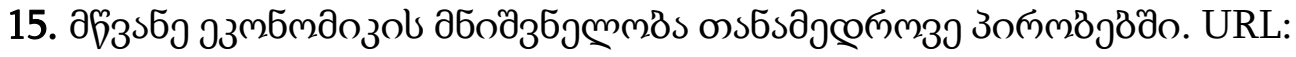
http://www.bec.ge/images/doc/green\%20economy.pdf

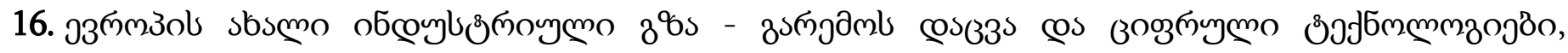
06.04.2020. URL: http://eugeorgia.info/ka/article/819/evropis-axali-industriuli-gza---garemos-dacva$\underline{\text { da-cifruli-teqnologiebi/ }}$ 


\title{
To identify priorities in science and technology
}

\author{
M. Matsaberidze 1 , J. Kerkadze ${ }^{2}$, I. Janelidze ${ }^{3}$, G. Jandieri ${ }^{4}$ \\ ${ }^{1}$ Georgian Technical University, Doctor of Chemistry, Professor Orcid ID: https://orcid.org/0000-0002-3228-1447 \\ ${ }^{2}$ Member-Corresponding of the Georgian Engineering Academy. Georgian Technical University; Faculty of Chemical \\ Technology and Metallurgy; Department of Environmental Engineering and Ecology; Ph. Doctor, Associate Professor; \\ e-mail:j.kerkadze@gtu.ge \\ ${ }^{3}$ Georgian Technical University: Faculty of Chemical Technology and Metallurgy, PhD in Chemistry, Professor (Assistant) \\ E-mail: i.janelidze@gtu.ge; Orcid ID: https://orcid.org/0000-0002-9961-7638 \\ ${ }^{4}$ Metallurgical Engineering and Consulting Ltd; Director, PhD (Technical Sciences), \\ E-mail: gigo.jandieri@gmail.com; Orcid ID: https://orcid.org/0000-0003-2976-1201
}

\section{Summary}

The sectoral priorities of science and technology determine the socioeconomic factors of the growth of states, which in turn provides for the system components of public safety.

The most important components of all sectoral priorities are synergistic-hybrid technologies, i.e. such technologies that are formed in the field of inter-sectoral competence.

To solve the problems of determining priorities in the field of synergistic-hybrid technology, the decisive function is the implementation of philosophical understanding of the problem, which is put forward as the main factor in decision making through the key components of sustainable development.

Keywords: Brain sciences; Mathematized world of science; Green economy; Green jobs; NBICtechnology; Transhumanism; Posthuman. 